\title{
Clinical and Morphological Aspects of High- intensity Focused Ultrasound (HIFU) Application in Breast Fibroadenoma Treatment
}

DOI: $10.21859 /$ mci-supp-112

\section{Keywords:}

High-intensity Focused Ultrasound (HIFU)

HIFU treatment

Benign Breast

Breast Fibroadenoma

Post-treatment Comparison

\author{
Syundyk Imankulov ${ }^{1}$, Zhazira Seidagliyeva ${ }^{2,}$, , Nurlan Zhampeissov ${ }^{3}$ \\ ${ }^{1}$ Astana Medical University Astana, Kazakhstan \\ * Corresponding author: Zhazira Seidagliyeva, Astana Medical University Astana, \\ Kazakhstan.E-mail:daken_89@mail.ru
}

\begin{abstract}
Introduction: Purpose of this study is to evaluate efficiency of HIFU method through morphocytological analysis and supervision during two-year post-treatment period, analyze relation between ablation power and morphological results.

Materials and Methods: The study involved 80 patients with breast fibroadenomas: 40 were HIFU-treated (main group) and others surgically (control). Morhpocytological analysis was carried after 1 day, 12 months and 24 months after treatment. For comparison analysis, all obtained results were used for statistical analysis with software STATISTICA and IBMSPSS. We conducted in-vitro investigations and morphocytological study with 3 circular cross-sections of fibroadenomas ablated by HIFU with 100W, 200W, 300W. All patients were surveyed by Questionnaire SF-36 checking physical functioning, level of pain, overall well-being, etc.

Results: No postoperative complications in main (HIFU) and 8 complications in control groups, 5 recurrences after 12-18 months were revealed in control group and 0 in main, inpatient stay duration was less by $52 \%$ in main group than in control group. Power ablation investigations show that $100 \mathrm{~W}$ ablation created initial changes related to coagulation necrosis, full coagulation necrosis observed at 200W and complete destruction of tissue at $300 \mathrm{~W}$. Intensity of pain by $44.7 \%$ and stimulated early resumption of functional activeness by $66.7 \%$.

Conclusions: HIFU therapy is new effective treatment method for breast fibroadenoma, that has less post-treatment complications and recurrences than surgical method, and may be used as an independent treatment of breast fibroadenoma.
\end{abstract}

